\title{
Progress in Super-Resolution from a Single Fluorescent Image Using the SUPPOSe Algorithm
}

Micaela Toscani ${ }^{1,4^{*}}$ and Sandra Martínez ${ }^{2,3,4}$

1. Departamento de Física, FIUBA, Buenos Aires, Argentina.

2. Departamento de Matemática, FCEyN, Buenos Aires, Argentina.

3. Instituto de Investigaciones Matemáticas Luis A. Santaló (IMAS).

4. Consejo Nacional de Investigaciones Científicas y Técnicas (CONICET).

* Corresponding author: mtoscani@fi.uba.ar

The SUPPOSe algorithm recently presented [1] relies in assuming that the image source can be described by an incoherent superposition of point sources of equal intensity and finding the number and position of such sources. The retrieval is performed using a genetic algorithm that finds an adequate fit to the measured signal. In this work we describe the recent advances in the implementation of the algorithm to gain resolution and remove artifacts arising from sources near the edge of the frame. The algorithm was slightly modified removing the invariant used before given by the product of the intensity of the sources times the number of sources, and replacing it by a new invariant given by the total intensity within the frame, thus allowing the location of sources outside the frame area.

As an example in the figure 1 BPA endothelial cells F-Actin labeled with Alexa Fluor 488 Phalloidin are shown: (a). as measured (b) reconstructed image using SUPPOSe. The improvement in resolution is obvious, and the presence of entangled fibers is unveiled from an image appearing in the raw data as a single strand. 


\section{References:}

[1] Sandra Martínez, Micaela Toscani and Oscar E. Martínez, Super-resolution method for a single wide field image deconvolution by superposition of point sources, J. Microscopy, https:// doi.org/10.1111/jmi.12802 (07 May 2019).
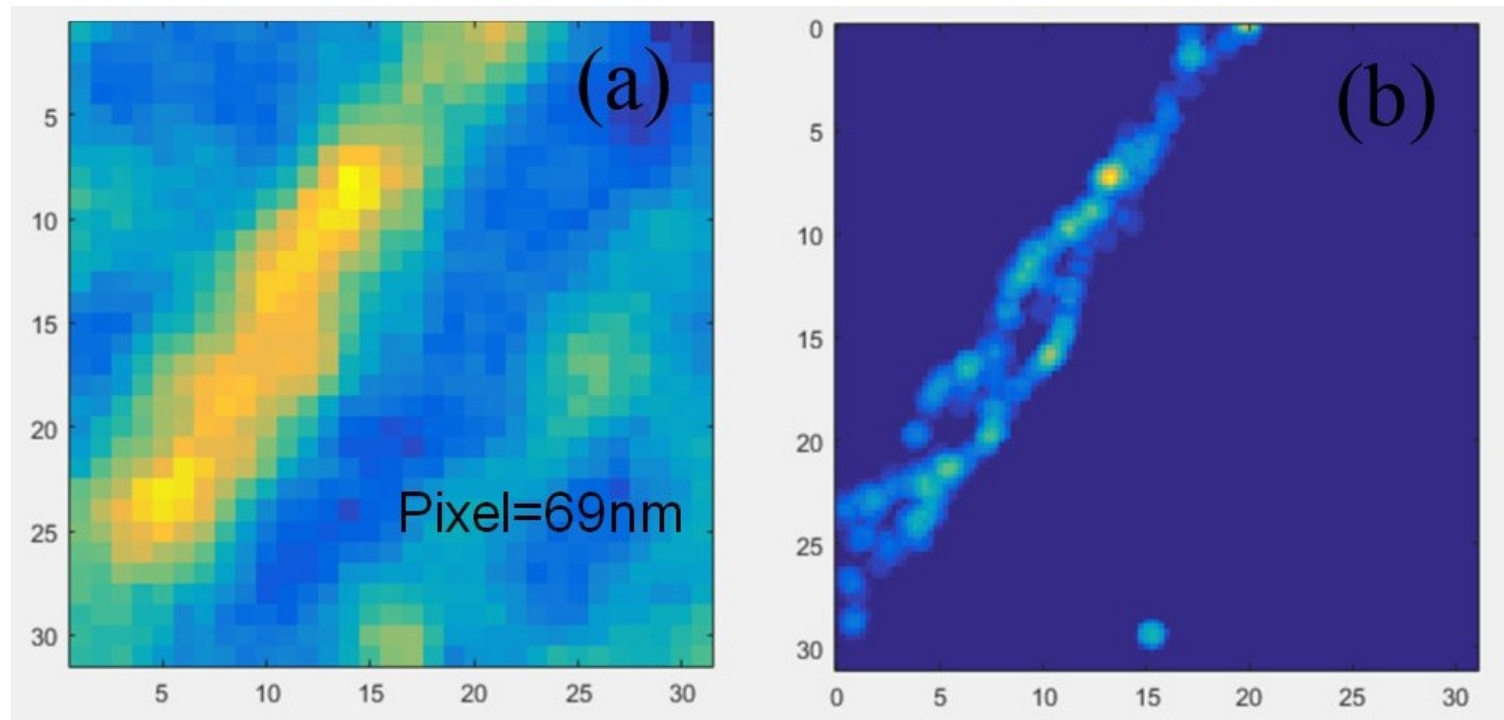

Figure 1. BPA endothelial cells F-Actin labeled with Alexa Fluor 488 Phalloidin are shown: (a) as measured (b) reconstructed image using SUPPOSe. 